\title{
Metafluid Dynamics as a Gauge Field Theory
}

\author{
A. C. R. Mendes ${ }^{a}$, C. Neves ${ }^{b}$, W. Oliveira ${ }^{b}$, and F.I. Takakura ${ }^{b}$ \\ ${ }^{a}$ Centro Brasileiro de Pesquisas Físicas, Rio de Janeiro, 22290-180, RJ, Brasil, \\ ${ }^{b}$ Departamento de Física, ICE, Universidade Federal de Juiz de Fora, \\ 36036-330, Juiz de Fora, MG, Brasil
}

Received on 11 September, 2002. Revised version received on 2 February, 2003.

\begin{abstract}
In this paper, the analog of Maxwell electromagnetism for hydrodynamic turbulence, the metafluid dynamics, is extended in order to reformulate the metafluid dynamics as a gauge field theory. That analogy opens up the possibility to investigate this theory as a constrained system. Having this possibility in mind, we propose a Lagrangian to describe this new theory of turbulence and, subsequently, analyze it from the symplectic point of view. From this analysis, a hidden gauge symmetry is revealed, providing a clear interpretation and meaning of the physics behind the metafluid theory. Also, the geometrical interpretation to the gauge symmetries is discussed.
\end{abstract}

\section{Introduction}

The understanding of hydrodynamic turbulence is an important problem for nature science, from both, theoretical and experimental point of view, and has been investigated intensively $[1,2,3]$ over the last century, but a deep and fully comprehension of the problem remains obscure.

Over the last years, the investigation of turbulent hydrodynamics has experienced a revival since turbulence has became a very fruitful research field for theoreticians, that study the analogies between turbulence and field theory, critical phenomena and condensed matter physics $[4,5,6,7,8$, $9,10,11,12]$, renewing the optimism to solve the turbulence problem.

The dynamics of turbulent viscous fluid is expressed by the Navier-Stokes (NS) equations of motion[1], which in vectorial form are given by

$$
\frac{\partial \overrightarrow{\mathrm{u}}}{\partial t}=-\overrightarrow{\mathrm{u}} . \nabla \overrightarrow{\mathrm{u}}-\frac{1}{\rho} \nabla p+\nu \nabla^{2} \overrightarrow{\mathrm{u}}
$$

or

$$
\frac{\partial \overrightarrow{\mathrm{u}}}{\partial t}=-\overrightarrow{\mathrm{w}} \times \overrightarrow{\mathrm{u}}-\nabla\left(\frac{p}{\rho}+\frac{\mathrm{u}^{2}}{2}\right)+\nu \nabla^{2} \overrightarrow{\mathrm{u}},
$$

where $\overrightarrow{\mathrm{u}}(\vec{x}, t)$ is the velocity field, $\overrightarrow{\mathrm{w}}(\vec{x}, t)$ the vorticity field, $p(\vec{x}, t)$ is the pressure, $\rho$ the density and $\nu$ the kinematic viscosity.

The equation of continuity reduces to the requirement that the velocity field is divergenceless for incompressible fluids, i.e,

$$
\nabla \cdot \overrightarrow{\mathrm{u}}=0,
$$

which are the flows we are interested in this paper. In this context, the hydrodynamic turbulence has attracted an enormous interest due to the universal characteristics stressed by an incompressible fluid with high Reynolds numbers in the fully developed turbulent regime. The Reynolds number,
$R \equiv L U / \nu$ (where $L$ is the integral length-scale of the largest eddies and $U$ is a characteristic large-scale velocity), measures the competition between convective and diffusive processes in an incompressible fluid described by the NS equations. The term $\overrightarrow{\mathrm{u}} . \nabla \overrightarrow{\mathrm{u}}$ is of the order of magnitude of $U^{2} / L$ and the quantity $\nu \nabla^{2} \overrightarrow{\mathrm{u}}$ is of the order of magnitude of $\nu U / L^{2}$. So, the ratio of the two is just the Reynolds number. Hence the term $\vec{u} . \nabla \vec{u}$ may be neglected if the Reynolds number is small, and the NS equations become the diffusion equation, $\frac{\partial \overrightarrow{\mathrm{u}}}{\partial t}=\nu \nabla^{2} \overrightarrow{\mathrm{u}}$, where the viscosity $\nu$ plays the role of the diffusion coefficient. So, as we are interested in treating the turbulence flow, we must consider high Reynolds number. In view of this, the incompressible fluid flow assumes high Reynolds numbers when the velocity increases and, consequently, the solution for eqn.(2) becomes unstable and the fluid switches to a new regime of a very complex motion with the velocity varying almost randomly and without any noticeable order. To discover the laws describing what exactly is going on with the fluid in this turbulent regime is very important to both theoretical and applied science.

Recently, Marmanis[13] has proposed an alternative approach to treat fluid turbulence. Based on the analogy between Maxwell electromagnetism and turbulent hydrodynamics, he describes the dynamical behavior of average flow quantities in incompressible fluid flows with high Reynolds numbers exactly as is done to obtain the macroscopic electromagnectic fields [14]. This average is obtained applying the spatial filtering method proposed by Russakoff[14, 15]. In this method, the spatial average of a function $A(\vec{x}, t)$ with respect to a test function $f(\vec{x})$ will be defined in terms of a convolutional integral given by

$$
\langle A(\vec{x}, t)\rangle=\int f\left(\vec{x}^{\prime}\right) A\left(\vec{x}-\vec{x}^{\prime}, t\right) \mathrm{d}^{3} x^{\prime},
$$


where $f(\vec{x})$ is real, positive definite in some neighborhood of $\vec{x}=\overrightarrow{0}$, and normalized to unity over all space. Moreover, in order to preserve directional characteristics, we can consider the class of functions $f(\vec{x})$ that are isotropic in space.

In this theory, metafluid dynamics, the vorticity $(\overrightarrow{\mathrm{w}}=$ $\nabla \times \overrightarrow{\mathrm{u}})$ and Lamb vector $(\overrightarrow{\mathrm{l}}=\overrightarrow{\mathrm{w}} \times \overrightarrow{\mathrm{u}})$ are recognized as the kernel of this dynamical theory of turbulence. In our paper, we make a further investigation of the physical contents present in that theory, in order to furnish a better understanding of turbulence.

To extract the physical meaning of the metafluid dynamics, we extend the Marmanis analogy in order to propose an appropriate Lagrangian governing the dynamics of incompressible fluid flows. From this Lagrangian and using the symplectic method[16], the physics behind the metafluid theory is discussed.

In order to make this work self-consistent, we have organized this paper as follows. In Section 2, a brief review of the metafluid dynamics is presented as well as the pertinent physical quantities and notation. In Section 3, the Lagrangian approach for the metafluid dynamics is proposed and some considerations about the dissipation rate of energy is discussed. In Section 4, the new description for the metafluid dynamics is studied using the symplectic method, which leads to unveil the gauge symmetry. Further, we scrutinize the symmetry of the model and show that the gauge invariance is only preserved in some limits, called inertial range, and also we identify the physical quantities that are gauge invariant into this region. In Section 5, the geometric interpretation of the gauge symmetry is given and discussed. In Section 6, we stress our conclusion and final discussions. We added an appendix with a brief review of the simplectic formalism.

\section{Metafluid dynamics}

The problem of turbulence is to find the averaged properties from the solutions of NS equations under the constraint of the incompressibility condition, eqn.(3), forming a system of coupled nonlinear partial differential equations. Due to this, it is a difficult task to get a common feature of averaged nonlinear equations, because nonlinearity introduces higher order momenta of fluctuation, what is known as closure problem. To overcome this problem, Marmanis[13] proposed an approximate theory of turbulence based on the analogy between Maxwell electromagnetism and turbulent hydrodynamics, where the equations governing the dynamic variables become linear and the nonlinearities appear as sources of turbulent motion. In this picture, one constructs a system of equations written in terms of the average values of the vorticity $(\overrightarrow{\mathrm{w}})$ and Lamb vector $(\overrightarrow{\mathrm{l}})$, instead of the average values of $\overrightarrow{\mathrm{u}}$ and $p$. Essentially, it turns the turbulence closure problem into a study of turbulent sources for different geometries of interest.

In this scenario, the study of average quantities of an

\footnotetext{
${ }^{1}$ This point is well discussed in Ref.[13].
}

incompressible fluid at the fully developed regime is proposed, leading to a system where the average fields show up in a continuum inter-relation and respond as waves to the turbulent sources. To do so, the Lamb vector and the vorticity should be taken as the kernel of the turbulent dynamics rather through velocity and vorticity fields or velocity and pressure fields. Then whatever parts that cannot be explicitly expressed as a function of $\overrightarrow{\mathrm{w}}$ or $\overrightarrow{\mathrm{l}}$ only, are gathered and treated as source terms. This is done by introducing the concepts of turbulent charge $(\mathrm{n})$ and turbulent current $(\vec{j})$. The turbulent charge is connected with the Bernoulli energy function,

$$
\Phi(\vec{x}, t)=\frac{p}{\rho}+\frac{\mathrm{u}^{2}}{2},
$$

through the relation

$$
\mathrm{n}(\vec{x}, t)=-\nabla^{2} \Phi
$$

In this formalism, the equations of motion describing the behavior of the hydrodynamic turbulence are

$$
\begin{aligned}
& \nabla \cdot \overrightarrow{\mathrm{w}}=0, \\
& \frac{\partial \overrightarrow{\mathrm{w}}}{\partial t}=-\nabla \times \overrightarrow{\mathrm{l}}+\nu \nabla^{2} \overrightarrow{\mathrm{w}}, \\
& \nabla \cdot \overrightarrow{\mathrm{l}}=\mathrm{n}(\vec{x}, t), \\
& \frac{\partial \overrightarrow{\mathrm{l}}}{\partial t}=\mathrm{u}^{2} \nabla \times \overrightarrow{\mathrm{w}}-\vec{j}(\vec{x}, t)+\nu \nabla \mathrm{n}(\vec{x}, t)-\nu \nabla^{2} \overrightarrow{\mathrm{l}},
\end{aligned}
$$

where the turbulent current $(\vec{j})$ is given by

$$
\vec{j}(\vec{x}, t)=\vec{u} n+\nabla \times(\vec{u} \cdot \vec{w}) \vec{u}+\vec{w} \times \nabla\left(\Phi+\vec{u}^{2}\right)+2(\vec{l} . \nabla) \vec{u} .
$$

After the average process ${ }^{1}$ are written as

$$
\begin{aligned}
& \nabla \cdot \vec{w}=0, \\
& \frac{\partial \vec{w}}{\partial t}=-\nabla \times \vec{l}+\nu \nabla^{2} \vec{w}, \\
& \nabla \cdot \vec{l}=n(\vec{x}, t), \\
& \frac{\partial \vec{l}}{\partial t}=c^{2} \nabla \times \vec{w}-\vec{J}(\vec{x}, t)+\nu \nabla n(\vec{x}, t)-\nu \nabla^{2} \vec{l},
\end{aligned}
$$

where $c^{2}=\left\langle u^{2}\right\rangle$ is the spatial averaged squared velocity, $\vec{w}$ and $\vec{l}$ are defined as the averages of $\vec{l}$ and $\overrightarrow{\mathrm{w}}$, while the sources $\vec{J}$ and $n$ are averages of $\vec{j}$ and n, respectively. As showed by Marmanis[13], taking $c^{2}=\left\langle u^{2}\right\rangle$ does not change significantly the pattern of velocity fields one get using eqn.(7) or eqn.(9). From now on, we refer to the above equations for averaged quantities as metafluid dynamics equations of motion.

Now, with the purpose to implement the analogy between the set of eqn.(9) with the Maxwell equations with sources in vacuum[14], note that these set of equations are identical if $\vec{B}$ (magnetic field) corresponds to $\vec{w}$ and $\vec{E}$ (electric field) corresponds to $\vec{l}$. Furthermore, the analogy can be extended in order to include the potentials as well. In particular, the comparison suggests that the vector potential corresponds to the velocity field $\vec{u}$ and the scalar potential to the Bernoulli energy function $\phi$. 
Despite of all resemblance between hydrodynamic turbulence and electromagnetic theory, there is a conceptual difference in the identification of the physical entities. In the classical electromagnetism, the physical fields are the electric and magnetic fields while the potentials are just mathematical artifices. Oppositely, in the metafluid dynamics, the potentials are the entities which have physical significance.

\section{Lagrangian approach}

From the geometric point of view and using the Lie algebra, Arnold [17] showed that Euler flow can be described in the Hamiltonian formalism in any dimension. This has a lot of interesting consequences for fluid mechanics and has been studied intensively[18, 19]. However, it is not quite obvious that this process can be used when viscosity is taken in account. It is in this scenario where the metafluid dynamics births, revealing a way to find a Hamiltonian formalism for a turbulent flow with viscosity.

In the classical electromagnetism, the Lagrangian density can be written as the difference between the square of the electric and magnetic fields, as follows,

$$
\mathcal{L}=\frac{1}{2}\left(\vec{E}^{2}-\vec{B}^{2}\right)
$$

Using the analogy established between electromagnetism and turbulence, one can write down the Lagrangian density for the theory of turbulence as

$$
\mathcal{L}=\frac{1}{2}\left(\vec{l}^{2}-c^{2} \vec{w}^{2}\right)
$$

where we define the averaged Lamb vector $\vec{l}$, as

$$
\vec{l}=-\frac{\partial \vec{u}}{\partial t}-\nabla \phi+\nu \nabla^{2} \vec{u}
$$

with $\vec{u}=\langle\overrightarrow{\mathrm{u}}\rangle$ and $\phi=\langle\Phi\rangle$.

So, we can also write the above Lagrangian density in terms of velocity field and the Bernoulli energy function, named "potentials of theory", as

$$
\mathcal{L}=\frac{1}{2}\left(-\nabla \phi-\frac{\partial \vec{u}}{\partial t}+\nu \nabla^{2} \vec{u}\right)^{2}-\frac{1}{2} c^{2}(\nabla \times \vec{u})^{2} .
$$

It is easy to see that this Lagrangian density gives us the equations of motion (9) for the homogeneous case (no sources).

Applying Euler-Lagrange equations for $\phi$ and $\vec{u}$, respectively, one gets

$$
\nabla \cdot(-\nabla \phi-\dot{\vec{u}}+\nu \nabla \vec{u})=0
$$

using eqn.(12), one can see that eqn.(14) can be written as

$$
\nabla \cdot \vec{l}=0 .
$$

For $\vec{u}$, we get

$$
\frac{\partial}{\partial t}\left(-\nabla \phi-\dot{\vec{u}}+\nu \nabla^{2} \vec{u}\right)=c^{2}(\nabla \times \nabla \times \vec{u})-\nu \nabla^{2}\left(-\nabla \phi-\dot{\vec{u}}+\nu \nabla^{2} \vec{u}\right) .
$$

Again, because of eqn.(12) and using $\vec{\omega}=\nabla \times \vec{u}$, the above equation can be written as

$$
\frac{\partial \vec{l}}{\partial t}=c^{2} \nabla \times \vec{\omega}-\nu \nabla^{2} \vec{l}
$$

Those equations of motion we got from the Lagrangian density (13) are the two last equations for the metafluid dynamics with no turbulent sources. The other two equations are obtained directly from $\vec{\omega}$ and $\vec{l}$ definitions. Taking the divergence of $\vec{\omega}$, we get the first of eqn.(9) and to get the second one, take the curl of eqn.(12).

Now, let us consider the case when sources do not vanish. In this case, the sources appear in the equations of motion and, as a consequence, an interaction Lagrangian, defined as

$$
\mathcal{L}_{i n t}=\vec{J} . \vec{u}-n \phi-\nu \vec{u} . \nabla n,
$$

which contains a viscous correction term, is added to the Lagrangian (13), providing the total Lagrangian density,

$$
\mathcal{L}=\frac{1}{2}\left(-\nabla \phi-\frac{\partial \vec{u}}{\partial t}+\nu \nabla^{2} \vec{u}\right)^{2}-\frac{1}{2} c^{2}(\nabla \times \vec{u})^{2}+\vec{J} . \vec{u}-n \phi-\nu \vec{u} . \nabla n
$$

There is no doubt that the metafluid dynamics can produce some interesting results. Hence, let us show that the equations of motion for viscous fluid, given in eqn.(9), can be found from the Euler-Lagrange equations, where the velocity field $(\vec{u})$ and the Bernoulli energy function $(\phi)$ are the canonical variables. From the Lagrangian density, eqn.(19), NS equations (2) are obtained in order to demonstrate that the physical contents of turbulence in the NS equations description are also present in our construction. This is reasonable, once in the process of averaging, actually, the test 
function used is supposed to cancel just the short distance fluctuations of velocity fields, so all the physical contents due to the random characteristic of turbulent flow observed in the fully developed turbulence, where the scale invariance is expected to exist, should not depend on short distance interactions. The conjugated momenta of velocity field is computed as

$$
\vec{\pi}(\vec{x}, t)=\frac{\delta \mathcal{L}}{\delta \dot{\vec{u}}(\vec{x}, t)}=\frac{\partial \vec{u}}{\partial t}+\nabla \phi-\nu \nabla^{2} \vec{u}=-\vec{l}(\vec{x}, t),
$$

and using (12), we get the NS equations, namely,

$$
\frac{\partial}{\partial t} \vec{u}(\vec{x}, t)=-\vec{l}(\vec{x}, t)-\nabla \phi(\vec{x}, t)+\nu \nabla^{2} \vec{u}(\vec{x}, t)
$$

where $\vec{l}(\vec{x}, t)=\vec{w} \times \vec{u}$.

One of the simplest consequences of the NS equations (2), modified by the addition of the external force $\vec{f}$, may be obtained by taking its scalar product with $\vec{u}$ and integrating the result over the space coordinate. The result is the relation

$$
\frac{\mathrm{d}}{\mathrm{d} t} \int \frac{1}{2} \mathrm{u}^{2}=-\frac{\nu}{2} \int(\nabla \overrightarrow{\mathrm{u}})^{2}+\int \vec{f} \cdot \overrightarrow{\mathrm{u}},
$$

which expresses the energy balance: the time derivative of energy on the right hand side is equal to the difference of the injection rate $\int \vec{f} \cdot \vec{u}$ and the dissipation of energy rate $\frac{\nu}{2} \int(\nabla \overrightarrow{\mathrm{u}})^{2}$. Taking averages in the stationary state, we obtain

$$
\langle\overrightarrow{\mathrm{u}} . \vec{f}\rangle=\left\langle\frac{\nu}{2}(\nabla \overrightarrow{\mathrm{u}})^{2}\right\rangle \equiv \epsilon,
$$

that is the equality of the (intensive) mean injection and the mean dissipation rates of energy. The energy injection takes place at the distances of order of the integral scale by induction of big scale $L$ eddies. According to the picture of the turbulent flow proposed in 1922 by Richardson [20], the big eddies induce still smaller eddies and so on transferring energy from large to small distance scales. This process should not lead to a loss of energy until sufficiently small distance scales, say, smaller than $\eta$ (dissipative scales of Kolmogorov[3]), are reached. On scales smaller than $\eta$, the dissipative term $\nu \nabla^{2} \overrightarrow{\mathrm{u}}$ of the NS equation becomes important.

\section{Symplectic analysis}

In the last sections, the analogy between turbulent hydrodynamics and Maxwell electromagnetism was explored and a gauge field theory to describe the turbulent fluid, called metafluid theory, was proposed, suggesting that this theory can be analyzed as a constrained system. Both systems are characterized in phase space by the presence of some functions that depend on the coordinates and canonical momenta, denominated constraints, which restrain the dynamics of the model. There are some methods to handle constrained systems, however we analyze the metafluid theory $(\nu \neq 0)$ from the symplectic point of view[16]. In the appendix we present the symplectic formalism for completeness.

To implement the symplectic formalism, the Lagrangian density (19) will be rewritten in its first-order form, given by,

$$
\mathcal{L}^{(0)}=\vec{\pi} \cdot \dot{\vec{u}}-U^{(0)}
$$

where the canonical momenta and the zeroth-iterative symplectic potential $\left(U^{(0)}\right)$ are

$$
\begin{aligned}
& \vec{\pi}=\nabla \phi+\dot{\vec{u}}-\nu \nabla^{2} \vec{u} \\
& U^{(0)}=\frac{1}{2} \vec{\pi}^{2}-\vec{\pi} . \nabla \phi-\frac{1}{2} c^{2}(\nabla \times \vec{u})^{2}+\nu \vec{\pi} . \nabla^{2} \vec{u}-\vec{u} . \vec{J}+\phi n+\nu \vec{u} . \nabla n
\end{aligned}
$$

respectively.

From the set of symplectic variables $\xi_{i}^{(0)}=\left(u_{i}, \pi_{i}, \phi\right)$ and their respective one-form canonical momenta,

$$
\begin{aligned}
& a_{u_{i}}^{(0)}=\pi_{i}, \\
& a_{\pi_{i}}^{(0)}=0, \\
& a_{\phi}^{(0)}=0,
\end{aligned}
$$

the symplectic matrix is computed as

$$
f^{(0)}=\left(\begin{array}{ccc}
0 & -\delta_{i j} & 0 \\
\delta_{i j} & 0 & 0 \\
0 & 0 & 0
\end{array}\right) \delta(\vec{x}-\vec{y})
$$

which is singular, so, has a zero-mode $\tilde{v}^{(0)}=\left(v^{\phi}, 0,0\right)$. Contracting this zero-mode with the gradient of symplectic potential $U^{(0)}$, the following constraint appears,

$$
\Omega_{1}=\nabla \cdot \vec{\pi}(\vec{x})+n(\vec{x}) .
$$

In agreement with the symplectic formalism, this constraint is introduced into the Lagrangian through a Lagrange multiplier $\lambda$, namely,

$$
\mathcal{L}^{(1)}=\vec{\pi} \cdot \dot{\vec{u}}+\dot{\lambda} \Omega_{1}-U^{(1)}
$$

where the symplectic potential density is

$$
\begin{aligned}
& U^{(1)}=\left.U^{(0)}\right|_{\Omega_{1}=0} \\
& =\frac{1}{2} \vec{\pi}^{2}-\frac{1}{2} c^{2}(\nabla \times \vec{u})^{2}+\nu \vec{\pi} . \nabla^{2} \vec{u}-\vec{u} . \vec{J}+\nu \vec{u} . \nabla n .
\end{aligned}
$$

Considering now that the new set of symplectic variables is given in the following order, $\xi_{i}^{(1)}=\left(u_{i}, \pi_{i}, \lambda\right)$, we have 
the one-form canonical momenta as,

$$
\begin{aligned}
& a_{u_{i}}^{(1)}=\pi_{i}, \\
& a_{\pi_{i}}^{(1)}=0, \\
& a_{\lambda}^{(1)}=\nabla \cdot \vec{\pi}+n .
\end{aligned}
$$

The symplectic matrix $f^{(1)}$ is

$$
f^{(1)}=\left(\begin{array}{ccc}
0 & -\delta_{i j} & 0 \\
\delta_{i j} & 0 & \partial_{j}^{x} \\
0 & -\partial_{i}^{y} & 0
\end{array}\right) \delta(\vec{x}-\vec{y}),
$$

that is a singular matrix with a zero-mode given by,

$$
\tilde{v}^{(1)}=\left(v_{j}^{\vec{u}}, 0, v^{\lambda}\right)
$$

satisfying the following relation,

$$
v_{i}^{\vec{u}}-\partial_{i} v^{\lambda}=0
$$

Contracting the zero-mode $\left(\tilde{v}^{(1)}\right)$ with the gradient of the symplectic potential $\left(U^{(1)}\right)$, we get a new constraint,

$$
\begin{aligned}
\Omega_{2} & =\int \mathrm{d}^{3} \vec{x} v_{i}^{\vec{u}} \frac{\delta}{\delta u_{i}(\vec{x}, t)} \int \mathrm{d}^{3} \vec{y}\left\{-\frac{1}{2} c^{2}(\nabla \times \vec{u})^{2}+\nu \vec{\pi} \cdot \nabla^{2} \vec{u}+\nu \vec{u} \cdot \nabla n-\vec{u} . \vec{J}\right\} \\
& =\int \mathrm{d}^{3} \vec{x} v_{i}^{\vec{u}}(\vec{x}) J_{i}(\vec{x}) .
\end{aligned}
$$

Using (34), $\Omega_{2}$ becomes

$$
\Omega_{2}=\int \mathrm{d}^{3} \vec{x} v^{\lambda}(\vec{x}) \partial_{i} J_{i}(\vec{x})=-\int \mathrm{d}^{3} \vec{x} v^{\lambda}(\vec{x}) \frac{\partial}{\partial t} \nabla^{2} \phi(\vec{x}) .
$$

Note that the sympletic analysis opens up the possibility to investigate the metafluid dynamics in and out of the inertial range. In this paper, we analyse the physical contents behind the metafluid dynamics at the inertial range. The dynamics of metafluid theory out of inertial range will be investigated in a future work. It is important to regard that at the inertial range, despite of the existence of viscosity, there is no energy dissipation, then, the Bernoulli energy function $(\phi)$ is constant. Due to this, $\Omega_{2}$ vanishes and, consequently, the hidden gauge symmetry of the metafluid theory is revealed.

To finish the symmetry analysis, it is necessary to obtain the infinitesimal gauge transformation. In the symplectic context, the gauge transformations are generated by the zero-mode that does not produce a new constraint. In the present case, the zero-mode $\tilde{v}^{(1)}$, given by eqn.(33), does not generate a new constraint, consequently, it is the generator of the following infinitesimal gauge transformations,

$$
\begin{aligned}
\delta u_{i} & =\partial_{i} \epsilon \\
\delta \pi_{i} & =0, \\
\delta \phi & =-\dot{\epsilon}
\end{aligned}
$$

where $\phi \rightarrow \dot{\lambda}$ and $\epsilon$ is an infinitesimal time-dependent parameter. It is easy to verify that the Lagrangian (19) is invariant under these transformations,

$$
\begin{aligned}
\delta \mathcal{L} & =-\left(\partial_{i} \pi_{i}+n\right) \dot{\epsilon}+(-\dot{n}+\dot{n}) \epsilon+\left(\partial_{i} \pi_{i}+n\right) \nu \partial^{2} \epsilon \\
& =0 .
\end{aligned}
$$

However, for the Hamiltonian below,

$$
\mathcal{H}=\frac{1}{2} \vec{\pi}^{2}-\vec{\pi} \cdot \nabla \phi-\frac{1}{2} c^{2}(\nabla \times \vec{u})^{2}
$$

$$
+\nu \vec{\pi} . \nabla^{2} \vec{u}-\vec{u} . \vec{J}+\phi n+\nu \vec{u} . \nabla n
$$

we get

$$
\delta \mathcal{H}=\epsilon \partial_{i} J_{i}=-\epsilon \nabla^{2} \frac{\partial}{\partial t} \phi .
$$

We can observe that the gauge invariance is only preserved at the inertial range, where the Bernoulli energy function is constant.

In order to obtain the Dirac brackets among the phase space fields, we have to fix the gauge symmetry. It is done introducing a gauge condiction into the kinetical sector of first-order Lagrangian through a Lagrange multiplier. So, we choose a gauge fixing term that satisfies the condition of incompressibility of fluid, namely,

$$
\chi=\nabla \cdot \vec{u} .
$$

In view of this, the twice iterated Lagrangian is obtained as

$$
\mathcal{L}^{(2)}=\vec{\pi} \cdot \dot{\vec{u}}+\dot{\lambda} \Omega_{1}+\dot{\eta} \chi-U^{(2)},
$$

where the symplectic potential density is

$$
U^{(2)}=\left.U^{(1)}\right|_{\chi=0}=\frac{1}{2} \vec{\pi}^{2}+c^{2} \vec{u} \cdot \nabla^{2} \vec{u}+\nu \vec{\pi} \cdot \nabla^{2} \vec{u}-\vec{u} . \vec{J} .
$$

Considering now that the new set of symplectic variables is given in the following order $\xi_{i}^{(2)}=\left(u_{i}, \pi_{i}, \lambda, \eta\right)$, we have

$$
\begin{aligned}
& a_{u_{i}}^{(2)}=\pi_{i}, \\
& a_{\pi_{i}}^{(2)}=0, \\
& a_{\lambda}^{(2)}=\nabla \cdot \vec{\pi}+n, \\
& a_{\eta}^{(2)}=\nabla \cdot \vec{u} .
\end{aligned}
$$


Then, the corresponding symplectic matrix is obtained as

$$
f^{(2)}=\left(\begin{array}{cccc}
0 & -\delta_{i j} & 0 & -\partial_{i}^{x} \\
\delta_{i j} & 0 & -\partial_{i}^{x} & 0 \\
0 & -\partial_{j}^{x} & 0 & 0 \\
-\partial_{j}^{x} & 0 & 0 & 0
\end{array}\right) \delta(\vec{x}-\vec{y}) .
$$

This matrix is nonsingular and, consequently, the corresponding inverse matrix can be determined after a straightforward calculation. From the inverse of $f^{(2)}$, the nonvanishing Dirac brackets among the phase space fields is automatically identified, namely,

$$
\left\{u_{i}(\vec{x}), \pi_{j}(\vec{y})\right\}^{*}=\left(\delta_{i j}-\frac{\partial_{i}^{x} \partial_{j}^{x}}{\nabla^{2}}\right) \delta(\vec{x}-\vec{y}) .
$$

The next step would be the quantization of this constrained theory. Using the well known canonical quantiza- tion rule $\left(\{,\}^{*} \rightarrow-i[],\right)$, we have,

$$
\begin{aligned}
& {\left[u_{i}(\vec{x}), u_{j}(\vec{y})\right]=\left[\pi_{i}(\vec{x}), \pi_{j}(\vec{y})\right]=0} \\
& {\left[u_{i}(\vec{x}), \pi_{j}(\vec{y})\right]=-i\left(\delta_{i j}-\frac{\partial_{i}^{x} \partial_{j}^{x}}{\nabla^{2}}\right) \delta(\vec{x}-\vec{y}) .}
\end{aligned}
$$

Once we have the canonical quantization rule, we can apply standard Quantum Field Theory techniques to find the generating functional and, consequently, the correlation functions and all physical quantities [21, 22] one wish. Despite given an prescription to quantize the metafluid dynamics, we will not discuss further this question. This subject is one of our present research.

In order to show the consistence of our formalism, let us obtain the equations of the Metafluid Dynamics from the Hamilton's equations. Using the Dirac brackets given above and the Hamiltonian given in (39), we have

$$
\begin{aligned}
\frac{\partial \vec{u}(\vec{x})}{\partial t} & =\int d^{3} \vec{y}\{\vec{u}(\vec{x}), \mathcal{H}(\vec{y})\}^{*} \\
& =\vec{\pi}(\vec{x})-\frac{\nabla(\nabla \cdot \vec{\pi}(\vec{x}))}{\nabla^{2}}-\nabla \phi(\vec{x})+\frac{\nabla\left(\nabla^{2} \phi(\vec{x})\right)}{\nabla^{2}}+\nu \nabla^{2} \vec{u}(\vec{x})-\nu \nabla(\nabla \cdot \vec{u}(\vec{x}))
\end{aligned}
$$

and

$$
\begin{aligned}
\frac{\partial \vec{\pi}(\vec{x})}{\partial t} & =\int d^{3} \vec{y}\{\vec{\pi}(\vec{x}), \mathcal{H}(\vec{y})\}^{*} \\
& =-\nu \nabla^{2} \vec{\pi}(\vec{x})+\nu \nabla(\nabla \vec{\pi}(\vec{x}))+\vec{J}(\vec{x}) \\
& +c^{2}\left(\nabla^{2} \vec{u}(\vec{x})-\nabla(\nabla \cdot \vec{u}(\vec{x}))\right)-\frac{\nabla(\nabla \cdot \vec{J}(\vec{x}))}{\nabla^{2}}
\end{aligned}
$$

Since the velocity field is divergenceless for incompressible fluids and $\nabla \cdot \vec{\pi}=-n=\nabla^{2} \phi$, from the first equation in (48)we get

$$
\vec{\pi}(\vec{x}, t)=\frac{\partial \vec{u}}{\partial t}+\nabla \phi-\nu \nabla^{2} \vec{u}=-\vec{l}(\vec{x}, t)
$$

which is the same result obtained in (20). Using (50), $\nabla . \vec{J}=-\nabla^{2} \dot{\phi}=0$ and (49), we have

$$
\frac{\partial \vec{l}(\vec{x})}{\partial t}=c^{2} \nabla \times \nabla \times \vec{u}(\vec{x})-\vec{J}(\vec{x}, t)+\nu \nabla n(\vec{x}, t)-\nu \nabla^{2} \vec{l}(\vec{x}),
$$

which is the fourth equation in (9). The first equation and the second equation in (9) are obtained taking the curl of (3) and (50), respectively. The third is obtained taking the divergence of (51).

\section{Geometrical aspects of the gauge formalism}

In this section, we give a geometrical interpretation to the gauge symmetry present on the metafluid theory (gauge field theory). The description of fundamental particle interaction, with the assistance of the gauge field theory, introduces ex- tra degrees of freedom into the theory, that manifests itself in the singular nature of the respective Lagrangian or the presence of first class constraint in the equivalent Hamiltonian formulation. In this case, the phase space is larger than the physical one, which is a hypersurface determined by the constraints of the theory. In this gauge invariant scenario, the gauge potentials form an overcomplete basis and the gauge fields, which can be connected by an infinitesimal transformation, describe the same physical state. Thus, the gauge potentials are separated into equivalence classes with respect to the gauge group action, where each one denotes an orbit in the gauge field configuration. Transitions along(vertical) the orbits correspond to pure gauge transfor- 
mations, consequently, these paths have no physical significance. Oppositely, perpendicular(horizontal) paths to the orbits describe the time evolution of the physical system, then, they are physically important to the theory. Hence, to find out the equations of motion for the physical fields consist in solving the problem of constructing the horizontal paths, which is computed just doing a correct definition of the metric in the orbit space. To do so, we follow process developed in [23], where the authors demonstrated that the physical (orbit) space is equipped with a natural projective metric.

In view of this, our task is to get a singular Lagrangian, dynamically equivalent to (19), to govern the metafluid theory. It is achieved eliminating the Bernoulli energy function $(\phi)$ from the Lagrangian (19). It can be done since this field has no dynamics, as demonstrated in the last section. To eliminate this field from (19), we use the Euler-Lagrange equation of motion for $\phi$, given by,

$$
\phi(\vec{x})=-\frac{\partial_{i} \dot{u}_{i}+n}{\partial^{2}}+\nu \partial_{i} u_{i}
$$

Bringing back this result into the Lagrangian (19) and rewriting it in terms of field components, we get

$$
\begin{aligned}
\mathcal{L} & =\frac{1}{2} \dot{u}_{i} M_{i j} \dot{u}_{j}+\left(\partial_{i} \dot{u}_{i}\right) \frac{n}{\partial^{2}}+\frac{1}{2} \frac{n^{2}}{\partial^{2}}-\nu\left(\partial^{2} \dot{u}_{i}\right) M_{i j} u_{j} \\
& +\frac{\nu^{2}}{2}\left(\partial^{4} u_{i}\right) M_{i j} u_{j}+\frac{1}{2} c^{2}\left(\partial^{2} u_{i}\right) M_{i j} u_{j}+u_{i} J_{i},(53)
\end{aligned}
$$

where the metric of configuration space $M_{i j}$, reads as

$$
M_{i j}=\delta_{i j}-\frac{\partial_{i} \partial_{j}}{\partial^{2}}
$$

being a singular matrix which has $\partial_{i}$ as eigenvectors with zero eigenvalues,

$$
\partial_{i} M_{i j}=0 \text {. }
$$

As the gauge orbits lay down by the eigenvectors, are vertical to the physical hypersurface defined by the projective metric $M_{i j}\left(M^{2}=M\right)$, the infinitesimal gauge transformation for the velocity potential can be computed as

$$
\delta u_{i}=\partial_{i} \varepsilon
$$

which was also obtained in symplectic context.

At this stage, we would like to make some comments about the geometry associated to the gauge symmetry. From the Lagrangian (53), we identify a singular projective metric which defines a physical surface (orbit space) of the metafluid theory. Since this metric does not depend on the phase space fields, the curvature tensor is null, therefore, the orbit space is flat.

Now, we investigate the metafluid theory described by the Lagrangian (53) using the projector method[24]. From eqn.(53), the canonically conjugated momenta to the field $u_{i}$ are computed as

$$
\pi_{i}=M_{i j} \dot{u}_{j}-\frac{\partial_{i} n}{\partial^{2}}-\nu M_{i j} \partial^{2} u_{j}
$$

Contracting these canonical momenta with the eigenvectors $\partial_{i}$, the primary constraint is determined as

$$
\chi \equiv \partial_{i} \pi_{i}+n
$$

which agrees with the results obtained in the symplectic analysis.

From the Lagrangian (53), the Euler-Lagrange vector is obtained as

$$
E_{k}=M_{k j} \ddot{u}_{j}-c^{2} M_{k j} \partial^{2} u_{j}-J_{k}-\frac{\partial_{k} \dot{n}}{\partial^{2}}-\nu^{2} M_{k j} \partial^{4} u_{j} .
$$

Using the projector method[24], the equations of motion that satisfy the constraints are projected by the singular metric, namely,

$$
M_{i k} E_{k}=0
$$

and, then, we get the wave equation for the velocity[13], given by,

$$
\begin{aligned}
& M_{i j} \ddot{u}_{j}-c^{2} M_{i j} \partial^{2} u_{j}-M_{i j} J_{j}-\nu^{2} M_{i j} \partial^{4} u_{j}=0 \\
& \ddot{u}_{i}^{\perp}-c^{2} \partial^{2} u_{i}^{\perp}-J_{i}^{\perp}-\nu^{2} \partial^{4} u_{i}^{\perp}=0
\end{aligned}
$$

where the velocity and current were redefined as $u_{i}^{\perp}=$ $M_{i j} u_{j}$ and $J_{i}^{\perp}=M_{i j} J_{j}$, respectively, and $\perp$ denotes the transverse fields (gauge invariant fields). This set of equations together with the constraint condition, eqn.(58), define the metafluid dynamics with transverse fields. Due to this, the incompressibility condition is satisfied automatically, namely,

$$
\begin{aligned}
\partial_{i} u_{i}{ }^{\perp} & =\partial_{i} M_{i j} u_{j} \\
\partial_{i} u_{i}{ }^{\perp} & =0 .
\end{aligned}
$$

Contracting the eigenvector $\partial_{i}$ with the Euler-Lagrange vector,

$$
\begin{gathered}
\partial_{k} E_{k}=\partial_{k}\left(M_{k j} \ddot{u}_{j}\right)-c^{2} \partial_{k}\left(M_{k j} \partial^{2} u_{j}\right) \\
-\partial_{k} J_{k}-\partial_{k} \frac{\partial_{k} \dot{n}}{\partial^{2}}-\nu^{2} \partial_{k}\left(M_{k j} \partial^{4} u_{j}\right)
\end{gathered}
$$

we get the continuity equation, given by,

$$
\partial_{k} E_{k}=\dot{n}+\partial_{k} J_{k}=0 .
$$

As $n=-\nabla^{2} \phi$, we obtain

$$
-\nabla^{2} \dot{\phi}+\partial_{k} J_{k}=0
$$

As our aim is to investigate the physical meaning of the metafluid dynamics at the inertial range where $(\dot{\phi}=0)$, we get

$$
\partial_{k} J_{k}=0
$$

Therefore, the current satisfies the divergenceless condition. 


\section{Conclusion}

In this paper, we proposed to investigate incompressible turbulent hydrodynamics in the context of metafluid dynamics in order to open up the possibility to apply all the machinery very well known in quantum field theory. This investigation was possible due to the analogy of incompressible turbulent hydrodynamics with Maxwell electromagnetism. Exploiting this analogy, the Dirac brackets were computed through the symplectic formalism, which revealed a hidden symmetry presents on the metafluid gauge theory, only preserved in inertial range. Afterward, the geometrical meaning of the gauge symmetry was given, showing that the orbit space is flat and, consequently, the divergenceless condition $(\nabla \cdot \vec{J}=0)$ is naturally obtained, at least in the inertial range. Subsequently, the wave equations for the transverse (horizontal) velocity field were computed, which was also obtained in [13], but written in terms of the velocity field, both horizontal (gauge invariant) and vertical (gauge variant) components.

\section{Acknowledgements}

This work was partially supported by $\mathrm{CNPq}$ and FAPEMIG. Three of us (A.C.R.M., F.I.T. and W.O.) thank to $\mathrm{CNPq}$ for the financial support and three of us (C.N., F.I.T. and W.O.) would like to thank FAPEMIG for partial support.

\section{A Symplectic formalism}

Faddeev and Jackiw [25] and Barcelos and Wotzasek [26] showed how to implement the constraints directly into the canonical part of the first-order Lagrangian once this method is applied to first order Lagrangians. In order to systematize the symplectic gauge formalism, a general noninvariant mechanical model that has its dynamics governed by a Lagrangian $\mathcal{L}\left(a_{i}, \dot{a}_{i}, t\right)$ (with $\left.i=1,2, \ldots, N\right)$ is considered, where $a_{i}$ and $\dot{a}_{i}$ are the space and velocities variables respectively. Notice that this consideration does not lead to lost of generality. In the symplectic method, the firstorder Lagrangian written in terms of the sympletic variables $\xi_{\alpha}^{(0)}\left(a_{i}, p_{i}\right)$ (with $\left.\alpha=1,2, \ldots, 2 N\right)$, is required

$$
\mathcal{L}^{(0)}=A_{\alpha}^{(0)} \dot{\xi}_{\alpha}^{(0)}-U^{(0)}
$$

where $A_{\alpha}^{(0)}$ is the one-form canonical momenta, $(0)$ indicates that it is the zeroth-iterative Lagrangian and, $U^{(0)}$, the sympletic potencial. This Lagrangian one-form gives rise to the Euler-Lagrange equations

$$
f_{\alpha \beta}^{(0)} \dot{\xi}_{\alpha}^{(0)}=\frac{\partial U^{(0)}}{\partial \xi_{\beta}^{(0)}},
$$

where

$$
f_{\alpha \beta}^{(0)}=\frac{\partial A_{\beta}^{(0)}}{\partial \xi_{\alpha}^{(0)}}-\frac{\partial A_{\alpha}^{(0)}}{\partial \xi_{\beta}^{(0)}},
$$

and whose solutions depend on the invertibility of $f_{\alpha \beta}^{(0)}$. Of course, this is impossible if there are (true) constraints involved. Since they would make this matrix singular. If $\left(f_{\alpha \beta}^{(0)}\right)^{-1}$ exists, then we can write

$$
\dot{\xi}_{\alpha}^{(0)}=\left(f_{\alpha \beta}^{(0)}\right)^{-1} \frac{\partial U^{(0)}}{\partial \xi_{\beta}^{(0)}} .
$$

After that, the sympletic tensor, eqn.(69), is computed. Since this sympletic matrix is singular, it has a zero-mode $\left(\nu^{(0)}\right)$ that generates a new constraint when contracted with the gradient of potencial, namely,

$$
\Omega^{(0)}=\nu_{\alpha}^{(0)} \frac{\partial U^{(0)}}{\partial \xi_{\alpha}^{(0)}} .
$$

Through a Lagrange multiplier $\lambda$, this constraint is introduced into the zeroth-iterative Lagrangian (67), generating the next one

$$
\begin{aligned}
\mathcal{L}^{(1)} & =A_{\alpha}^{(0)} \dot{\xi}_{\alpha}^{(0)}-U^{(0)}+\dot{\lambda} \Omega^{(0)} \\
& =A_{\alpha}^{(1)} \dot{\xi}_{\alpha}^{(1)}-U^{(1)}
\end{aligned}
$$

where

$$
\begin{aligned}
U^{(1)} & =\left.U^{(0)}\right|_{\Omega^{(0)}=0}, \\
\xi_{\alpha}^{(1)} & =\left(\xi_{\alpha}^{(0)}, \lambda\right) \\
A_{\alpha}^{(1)} & =A_{\alpha}^{(0)}+\lambda \frac{\partial \Omega^{(0)}}{\partial \xi^{(0)}} .
\end{aligned}
$$

The first-iterative sympletic tensor is computed as

$$
f_{\alpha \beta}^{(1)}=\frac{\partial A_{\beta}^{(1)}}{\partial \xi_{\alpha}^{(1)}}-\frac{\partial A_{\alpha}^{(1)}}{\partial \xi_{\beta}^{(1)}} .
$$

Since this tensor is nonsingular, the iterative process stops and the Diras brackets among the phase-space variables are obtained from the inverse matrix. On the other hand, if the tensor is singular, a new constraint arises and the iterative process goes on.

\section{References}

[1] L.D. Landau and E.M. Lifshits, Fluid Mechanics (Pergamon Press, Oxford, 1980).

[2] A.S. Monin and A.M. Yaglom, Statistical Fluid Mechanics: Mechanics of Turbulence (The MIT Press, Cambridge, 1971).

[3] U. Frisch, Turbulence: The Legacy of A.N. Kolmogorov, Cambridge University Press, Cambridge (1995).

[4] S.T. Bramwell, P.C.W. Holdsworth and J.F. Pinton, Nature 396, 552 (1998).

[5] V.S. L’vov, Nature 396, 519 (1998).

[6] V. Periwal, cond-mat/9602123 (1996).

[7] A.M. Polyakov, Nucl. Phys. B396, 367 (1993). 
[8] V. Gurarie, Field Theory and the Phenomenon of Turbulence, (Proc. Recent progress in statistical mechanics and quantum field theory, Los Angeles, USA)(1994).

[9] G. Eyink and N. Goldenfeld, Phys. Rev. E50, 4679 (1994).

[10] M. Nelkin, Phys. Rev. A9, 388 (1974).

[11] P.G. De Gennes, Fluctuation, Instability and Phase Transition, (Proc. NATO Adv. Study Inst., Geilo, Norway). T. Riste, ed. (Noordhoff, Leiden), series B (1975), p1.

[12] H.A. Rose and P.L. Sulem, J. of Phys. 39, 441 (1978).

[13] Haralambos Marmanis, Phys. Fluids 10, 1428 (1998); $\mathrm{PhD}$ these: Analogy between the Electromagnetic and Hydrodynamic Equations: Application to Turbulence, 2000, http://www.cfm.brown.edu/people/marmanis/.

[14] J.D. Jackson, Classical Electrodynamics (J. Willey, New York, 1983)

[15] G. Russakoff, Am. J. Phys. 38, 1188 (1970).

[16] L. Faddeev and R. Jackiw, Phys. Rev. Lett. 60, 1692 (1988); J. Barcelos Neto and C. Wotzasek, Mod. Phys. Lett. A7, 1737 (1992); Int. J. Mod. Phys. A7, 4981 (1992).

[17] V.I. Arnold, Mathematical Methods of Classical Mechanics, (Springer, New York, 1989).
[18] V.I. Arnold and B.A. Khesin, Ann. Rev. Fluid Mech. 24, 145 (1992).

[19] V. Zeitlin, J. Phys. A25, L171 (1992).

[20] L.F. Richardson, Weather prediction by numerical process (Canbridge University Press, Canbridge, 1922).

[21] C. Itzykson and J. B. Zuber, Quantum Field Theory (McGraw-Hill Inc., New York, 1980).

[22] M. Henneaux and C. Teitelboim, Quantization of Gauge Systems (Princeton University Press, Princeton, 1992).

[23] M. Grabiak, B. Muller and W. Greiner, Ann. Phys. (NY) 172 (1986) 213;

N. Ilieva, L. Litov, Proceedings, Selected topics in QFT and mathematical physics, Liblice (1989) 239-250.

[24] C. Amaral and P. Pitanga, Nuovo Cimento B25, 817 (1982); Rev. Bras. Fis., 12 (3), 473 (1982).

[25] L. Faddeev and R. Jackiw, Phys. Rev. Lett. 60, 1692 (1988).

[26] J. Barcelos Neto and C. Wotzasek, Mod. Phys. Lett. A7, 1737 (1992); Int. J. Mod. Phys. A7, 4981 (1992). 